\title{
Ear Structure Feature Extraction Based on Multi-scale Hessian Matrix
}

\author{
$\mathrm{Ma} \mathrm{Chi}^{1,2,3}$,Ban Xiaojuan*1 ${ }^{1}$, Wang Guosheng ${ }^{3}$ and Tian Ying ${ }^{2}$ \\ ${ }^{1}$ School of Computer \& Communication Engineering, University of Science and \\ Technology Beijing, Beijing, China \\ ${ }^{2}$ College of Software, University of Science and Technology LiaoNing, Anshan, \\ China \\ ${ }^{3}$ Beihai Yinhe Industry Investment Co.,Ltd., Beihai, China \\ E-mail:asmachi@126.com
}

\begin{abstract}
In this paper, a new ear anatomy feature edge extraction method based on Hessian matrix is proposed. Stable edge is obtained from principal curvature image across scale space. Firstly, the side face image that includes an ear is filtered and forms Gaussian pyramid. Secondly, the 2D gray image in the pyramid was regarded as a surface, maximum and minimum principal curvature and their direction were calculated by using Hessian matrix, and principal curvature image was formed. The characteristic of surface is that gray level changes in edge area is sharp and the curvature is larger compared to that of the smooth area. In accordance with this characteristic, automatic hysteresis thresholding based on curvature direction flow is used to segment curvature images. Lastly, combine different scale threshold images to get the feature edge image. The experiments demonstrate that extracted feature edge is smooth and connected. New method is robust to noise, and is sensitive to the weak edge, using Hausdorff distance as similarity measurement of two edge images can obtain above $96 \%$ recognition rate.
\end{abstract}

Keywords: Ear recognition, feature extraction, edge detection, Hessian matrix, principal curvatures

\section{Introduction}

As a branch of the biometric identification, ear recognition has received much more attention in recent years. Human ears have rich and stable structure that is preserved from childhood into old age. Ear recognition has the advantages of easy acquiring data, easy to accept, low-cost equipment, and it does not suffer from changes in facial expression, aging, psychological factors or cosmetics. Meanwhile, human ears have smaller the image size and then have a small amount of data processing, have more consistent color distribution [1]. Therefore ear recognition technology become a potential for development of biometric identification technology, gradually get the academics attention.

Ear recognition has an important step of accurately extracted feature vector that can fully characterize the features of the human ear. The existing feature extraction method of the human ear can basically be divided into two categories [2]: based on the algebraic feature [3-4] and expression vector based on structural features [5].

The former treats the whole human ear as a global description of algebraic or statistical methods to extract the global features. Such as PCA [6], ICA [7], LPP [8] local tangent space [9], 2DLDA [10], wavelet transform [11], and so on.

* Corresponding Author 
These methods generally require a large amount of calculation and a group of experimental parameters related to the specific environment. Furthermore, although these algebraic methods can grasp the difference between the images, they are inherently sensitive to pose, illumination, scale and occlusion, and their robustness is poor.

The latter extracts discrete local structural features, and its weight is usually the edge that ear contour or internal groove back formed. In fact, the most distinguishing ability in the outer ear image feature is the shape characteristics of the outer ear and the external ear anatomical features, like the helix, earlobe, tragus and triangular fossa. As shown in Figure 1, the correct extraction of these edges information can identify the key issues based on the structural characteristics of the human ear. The correct extraction of these edges information is the key problem based on the structural characteristics of the human ear identification.

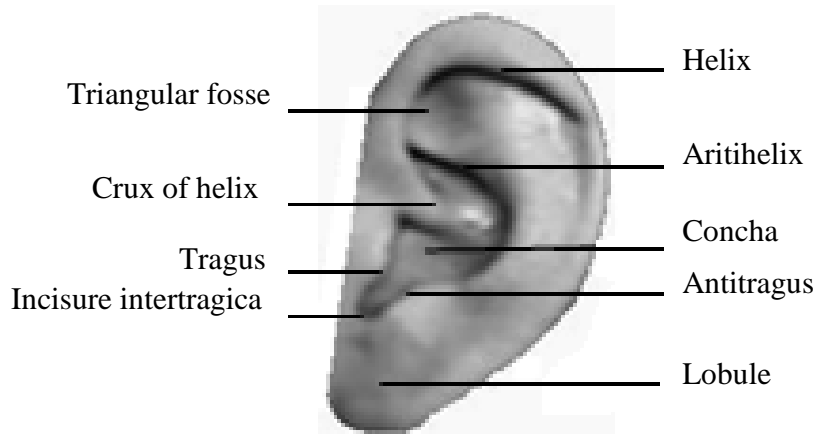

\section{Figure 1. Anatomy of Ear}

The method of extraction edge of ear structural features based on 2D grayscale image recognition is commonly used: Canny operator [12-14] feature edge extraction; Moreno[15] using Sobel operator feature edge detection; Choras [16] using the K-value method to extract the edge of the outer ear characteristics curve, not only extract the edge width, but also need to manually set the threshold $\mathrm{K}$, cannot meet the need of automatic processing; Mu Zhichun[17] use different methods to extract of the inner ear structural feature sedge edge and the outer ear shape features, and then combine them; literature [18] using the high-frequency component of the wavelet decomposition to extract edge information. These methods are generally small amount of calculation, intuitive sense of physical characteristics. But edge features and point features have direction and scale differences. If scale, rotation angle changes in the image, the extracted edge will produce errors, thereby increasing the false rejection rate.

Bustard and Nixon[19] proposed anear registration and recognition method by treating the ear as aplanar surface and creating a homograph transform using SIFT feature matches. They got rank-1 recognition rate of $92 \%$ for $30 \%$ occlusion from above and rank-1 recognition rate of $92 \%$ for $30 \%$ occlusion from left side on a set of 63 subjects from XM2VTS face profile dataset. Arbab-Zavar and Nixon[20] proposed a model-based approach for ear recognition. The model is a part-wise description of the ear derived by a stochastic clustering on a set of scale invariant features of the training set. The outer ear curves are further analyzed with log-Gabor filter. By fusing the model-based and outer ear metrics, they got a correct classification rate of $89.4 \%$ for $30 \%$ occlusion from above on the same set of 63 subjects. But these methods only tested ear occlusion from above or left side, other occlusion locations should be further discussed since the ears may be occluded at any locations in real scenarios.

The above method is basically using typical edge detection operator to extract the edge points, but in fact every kind of edge detection methods are proposed for a problem, no one can meet the needs of all practical applications. In 2D gray scale human ear image, the outer ear, neck and facial skin color very close, there are a lot of weak edges. Therefore, extracted the edge of the characteristics of the human ear using the above 
method, exists a certain error, the extracted edge line is discontinuous, or too much noise. Most image illumination requirements need to set the threshold value manually, cannot meet the automatic recognition of the human ear.

Based on this, the paper proposes a highly robust structural characteristic of the human ear edge detection method based on multi-scale Hessian matrix. Distribution in the gray space auricle image as a surface, due to the curved surface at the outer ear structure characterized by groove back change at a larger gray-scale variation of the position of curvature will be greater than the curvature of the gray-scale variation smoothness position. Therefore, by detecting a maximum value of the local curvature can obtain the auricle characterized edge curve.

After extracting characterized edge curve, we used thinning algorithm to obtain singlepixel edge of image. This edge image was a clear expression of original ear image. Then we regard the single-pixel feature curve edge as a key point set, using the Hausdorff distance as similarity measurement of two edge images for identification. Experiments in selected ear image database show that the method has ability to identify than other method. It has been enhanced recognition rate to image rotation, scale, light conditions change.

\section{Algorithm Description}

The distribution of the outer ear images in three-dimensional gray space can actually be seen as a curved surface. Surface expressed as $\mathrm{z}=\mathrm{f}(\mathrm{x}, \mathrm{y}),(\mathrm{x}, \mathrm{y})$ is gray image position information, $\mathrm{z}$ is grayscale. In the three-dimensional space coordinate system, the distribution of the image surfaces of the external ear is shown in Figure 2(b).

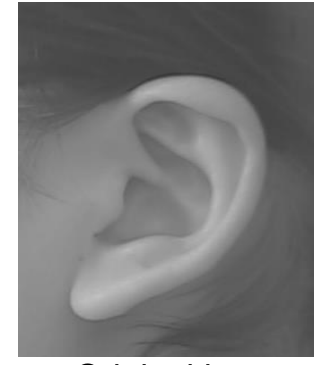

a. Original image

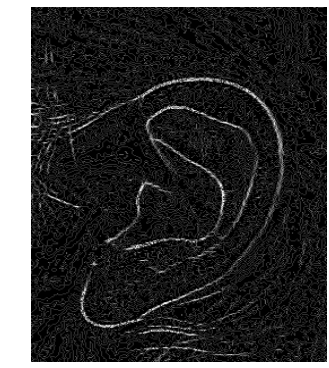

c. Curvature image

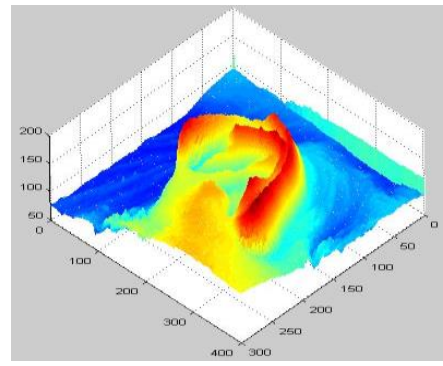

b. 3D surface image

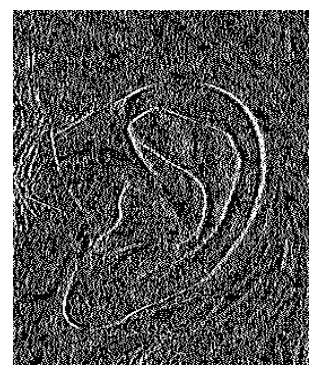

d. Grads image

Figure 2. Ear Surface Image

From figure 2(a) and 2(b), we can clearly see that the size of the gradation values and curved undulating correspondence relationship, the gradation is deeper, the undulation of the surface is greater. Further we can see also from the differential geometry knowledge, can calculate space surface curvature at any point, gray change of curvature of the point. Gray greater the change, the point of curvature is greater; if the gray-scale variation is relatively flat, the smaller curvature. Therefore, we can obtain by computing the principal curvature of each point on the method of the surfaces the main curvature of the gray-scale 
image corresponding image, as shown in Figure 2(c), and the structure of the edge of the main curvature image generated by this process is more clear than the usual gradientbased methods(Figure 2(d)) to obtain.

Because the light intensity can sometimes change the image acquisition process, the surface of the ear image brightness changes, and this change is manifested as changes in the overall average gray on the surface, that surface in three-dimensional space along the grayscale direction occurs on a pan, and did not change the relative position of the feature edges, i.e. no change of the undulation of the surface, does not affect the calculation result of the curvature. Therefore, the calculation of the curvature graph is not subject to the effects of changes in light intensity.

\subsection{The Principal Curvature Image Determined by Hessian Matrix}

1) Hessian matrix

A grayscale image as a gray surface, wherein the curve structure corresponding to the ridges and valleys of the surfaces. A point on the local shape of the surfaces can be characterized by the Hessian matrix to describe as followed:

$H(x)=\left[\begin{array}{ll}I_{x x}(x) & I_{x y}(x) \\ I_{x y}(x) & I_{y y}(x)\end{array}\right]$

Where: $I_{x x}, I_{\mathrm{xy}}$ and $I_{y y}$ is the second order partial derivatives of the image $I(x)$.

Hessian matrix is $n \times n$ real symmetric matrix, having $n$ real eigenvalues, herein $n=2 . n$ feature values of $H(x)$, the amplitude of the largest eigenvalue and its corresponding feature vector representing the intensity and direction of the maximum curvature point $x$, referred to as the main characteristic values and the main direction. Similarly, the amplitude of the smallest eigenvalue of the corresponding feature vector represents the intensity and direction of the minimum curvature of the point $x$, referred to as subcharacteristic value and the sub-direction, and two feature vectors orthogonal. This geometric meaning of the Hessian matrix has a wide range of applications. Such as the fingerprint image ridge extraction [21], the extraction of the center path [22] and so on. We will introduce it into the ear image characteristics of the edge of the extraction process.

2) Principal curvature image scale space

Calculate the eigenvalues and corresponding eigenvectors of the Hessian matrix of points $x$ on the surface, obtained the largest eigenvalue and corresponding eigenvectors, the principal curvatures images of $P(x)$ by the formula(2):

$P(x)= \begin{cases}\lambda_{\text {max }}(x) & \lambda_{\text {max }}(x)>0 \\ 0 & \lambda_{\text {max }}(x) \leq 0\end{cases}$

Where $P(x)$ is principal curvature of the pixels in the image, $\lambda_{\max }(x)$ is the largest eigenvalue of the Hessian matrix of the point $x$. We calculate the dark edge line under a bright background, therefore, the greatest feature value of the Hessian matrix for the point of the edge line is positive, and the characteristic value can be approximated for the point corresponding to the partial image gradation second direction derivative being extrema. But such direct principal curvature image $P$ to strike the characteristics of the edge effect is not ideal. Problem is: First, if the image has noise or interference, it will have large changes in the curvature, so obtained the edge points are instability; second, if the results do matching recognition when the scale changes of two images of the same ear, extraction characteristics of edge point becomes very accurate, and great class error will increase the false rejection rate(FRR) in the identification process. To this end, we calculate the principal curvature of image in scale space. 
First ear image scale expansion of 2 times as the initial image $I(x, y)$, and then the original image is continuous filtered by the Gaussian kernel function of the different scale to obtain a scale space image:

$L(x, y, \sigma)=G(x, y, \sigma) * I(x, y)$

$L$ is the image of scale space, $G(\mathrm{x}, y, \sigma)$ is Gaussian function, two-dimensional Gaussian function is defined as follows:

$G(x, y, \sigma)=\frac{1}{2 \pi \sigma^{2}} e^{-\left(x^{2}+y^{2}\right) / 2 \sigma^{2}}$

$\sigma$ is the scale factor of space, the smaller the value, that is, the smaller the scale, the less smooth of the characterization of the image, rich edge details of the image, the edge of the high positioning accuracy, but are susceptible to noise interference. In order to obtain a stable, high accuracy, the edge of the good features of noise resistance, detecting edge points in the image of the different scales, and then fused.

Therefore, the scale space of the main curvature of the image generation process (similar to the process with the SIFT[23-24]) can be described as:

1) Application (3) different scale Gaussian smoothing initial ear image $I$, initial scale factor $\sigma=\sqrt{2}$, step is $\sqrt{2}$, generate images $L 1 j, j=\{1,2,3,4,5\}$, from $L 11$ to $L 15$ formation of the first storey of the Sri Lanka pyramid image.

Table1. Ear Size and Select Seeds Amount

\begin{tabular}{cccc}
\hline Ear size & Smaller ear & Normal ear & le \\
\hline Ear radio & $E s \leq 30 \%$ & $30 \% \leq E s \leq 40 \%$ & $E s \geq 40 \%$ \\
Seeds count radio & $1.5 \%$ & $1.75 \%$ & $2 \%$
\end{tabular}

2) The down-sampled image $L 13$ to $L 21$ of the second layer of the first pyramid image, and to use it to form a second storey Adams pyramid size is half of the first layer image. Use with a layer of the same method as the second layer image of $L 21$ to $L 25$.

3 ) And so on to build the next layer of the pyramid image $L 31$ to $L 35$. The ear images smaller, so this creates only the three pyramids.

4) Calculate the principal curvature image $P i j$ on a scale image applications (2) with $i=1,2,3, j=1,2,3,4,5$.

5 ) Seeking the maximum corresponding points in each image with the adjacent upper and lower two image pyramid $M P i j(x)=\max (P i j-1(x), P i j(x), P i j+1(x))$, where $i$ represents layers, $\mathrm{j}$ represents the $j$ site principal curvature image. So that each layer can produce three images to form the main curvatures of the image pyramid.

\subsection{Automatic Thresholding}

Principal curvature image, in order to further contains only the goal edge without the noise edge clear and the edge of the binary image, the key issue is how to properly select the threshold into.

1) After hysteresis threshold

The study found a significant role in guiding the eigenvectors of the Hessian matrix of the determination of the edge points. Point located on the edge curve in a local area within the window has the same or similar views direction, i.e. the same direction as the flow. Based on this, we put forward a very strong robustness threshold scheme based on the direction of the feature points after stagnation, both can be connected to the relatively weak edge points, they can remove the noise edge. 
Define two thresholds: the high threshold $T h$ and low threshold $T l$, Hessian Matrix eigenvalue greater than Th the strong curvature response point, These points as the initial edge point. Initial edge points as seeds, seed point for expansion extensions, and then the neighborhood is greater than the low threshold $T l$ pixel contains come.

2) High threshold settings

Observed ear picture, although outer ear shape and inner ear structure curve is different, but the number of pixels constituting the edge curve relative to the auricle area is relatively fixed. The contour detection method we propose [25] is used for extracting outer ear contour and then the ear is segmented from side face. Extraction auricle and calculate the area, the set of seed points attributable auricle image area of 5\%. Because of the auricle of different sizes, according to the pinna area of the total image area ratio $(E s)$ of the auricle shown in Table 1, three kinds. Then select the ratio of the different sizes of ears on the seed points with reference to Table 1 .

The main characteristic values of the principal curvature image sorted intensity size accumulate statistics, when accumulated to select the ratio of the corresponding seed points, the corresponding eigenvalues strength is high threshold $T h$.

3) Low threshold settings

The low threshold value is a current pixel sub eigenvectors the acceptable support function from its neighboring pixels. A child window taken $w^{*} \mathrm{w}, \mathrm{w}$ and selection based on the size of the image, preferably in each window contains only a single strike section of edge, this article $w=20$. Comparing (the direction of the secondary direction of each seed pixels within the window and its neighbor pixel sub point the inner edge of the window times consistent with the direction, the main direction of the edge points consistent some opposite). Comparison of the method is to take the absolute value of the multiplication results of the current minimum feature vector of the Hessian matrix of the pixel and its neighboring pixels a minimum feature vector points (feature vector unit form). The larger the absolute value indicates the direction of the two vectors is more consistent, and when parallel to the two feature vectors, the point multiplication result absolute value is maximum; when its orthogonal minimum absolute. If the current pixel on all neighbor domain average point multiplication result sufficiently high, provided the ratio of the low and high threshold value of 0.3 (i.e. the low threshold value $T l=T h^{*} 0.3$ ); Otherwise, the low and high threshold value of the ratio as 0.8 (the lower threshold value $T l=T h * 0.8$ ), this result is achieved by a large number of data experiment drawn.

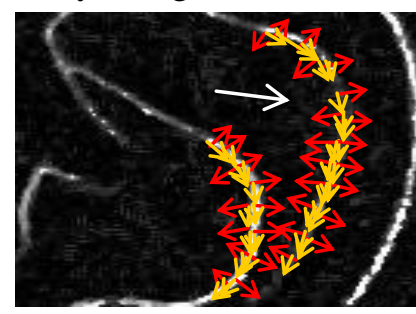

\section{Figure 3. How the Eigenvector Flow Helps Overcome Weak Edge}

Figure 3 illustrates the eigenvectors of the Hessian matrix of a weak edge structure. The red arrow is the major direction of the yellow arrow The direction, in order to improve the visual effect, we are 10 pixels indicates the strength of the vector. At the point indicated by the large white arrow, we see that the eigenvalue magnitudes are small and the ridge there is almost invisible. Nonetheless, the directions of the eigenvectors as indicated by the minimum feature are quite uniform, i.e. the point on the edge with the same direction of flow. Therefore, the edge points can be detected according to the direction of those weak. Thus, the effective threshold procedure based on the direction of the feature vector extraction features edge point. Due to changes in the direction of the point on the feature edges with continuity, while the background noise is random, and therefore most of the noise and interference can be removed by the above-mentioned 
threshold processing.

4) Stable edge point

Finally, in order to further improve the robustness of the algorithm, the main curvature after the image on the threshold processing each of a scale space, we image the results obtained on different scales the fusion treatment. Method is to retain only those on the scale of at least 3 can detect the characteristics of the edge point as the final edge. This means that we choose edge point by partially scale change is stable.

\section{Experimental Results and Analysis}

\subsection{Image Sources and Pretreatment}

At present, the ear images used in most works mainly come from three specific database: UND database collected by University of Norte Dame, UCR database connected by University of California at Riverside and USTB database collected by University of Science and Technology Beijing. We use the USTB ear image database I and image database II and ourselves human ear image database that we call it database III. Image database I contains 61 subjects, 3 ear images for per subject. Image database II contains 77 subjects, each subject has 4 ear images (frontal, left and right within $\pm 35^{\circ}$, and illumination variation). Image database III contains 15 subjects, each subject has 15 ear images ( 5 illumination variation and 10 pose variation, including rotation of $\pm 5^{\circ}$, $\pm 10^{\circ}, \pm 15^{\circ}, \pm 20^{\circ}, \pm 25^{\circ}$ ). We remove some poor quality images, and select 50 subjects from image database I and II respectively, select 10 subjects from Image database III. We select a total of 500 images as a small-scale sample database to do our experiment.

First, we range all the images in the image library into 256-color gray scale images. Because the main purpose of this method is to verify the robustness of the image light, rotation angle and scale change, there is no need to advance the image normalization .

Figure 4(h) show use this method, the partial result of the curvature characteristics of edge extraction. The whole process of the proposed algorithm is completely automatic and does not require any manual intervention. The results can be seen by the diagram of feature extraction, edge curve basic reaction of the anatomical features of the external ear, light and slight changes in the angle does not affect the ear image edge detection results.

In order to compare the performance of the algorithm, using the same hardware and software environment to achieve another five kinds of edge detection operators (Sobel operator, LoG operator, Canny operator, Priwitt operator as well as the literature [15] used $\mathrm{K}$-value of the algorithm). In addition, we also used the maximum class distance criterion threshold directly on the maximum principal curvature image binarization, feature edge map, which we call the curvature of the extreme value method. 

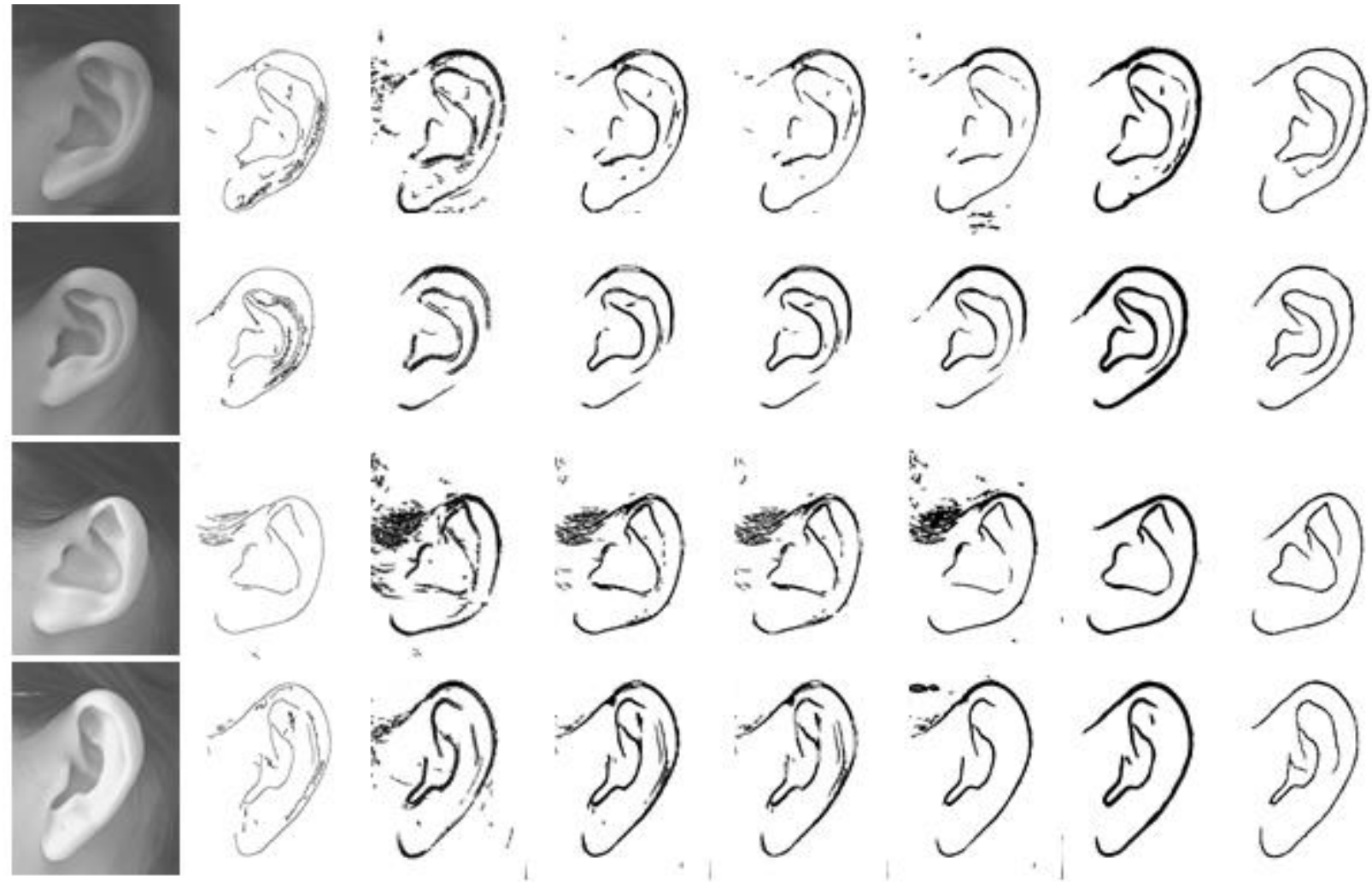

a.original image b.Canny operator c.LoG operator d.Priwit operator e.Sobel operator f.Lit[9] method g.curvature h.our method

Figure 4. Compare Results of our Method and Others

All experiments were tested on the same image library. Comparable with the parameters of the algorithm in edge detection using the best parameters of the overall effect of the test results to the experimental results. Since the proposed algorithm and several other algorithms handling time soon, is negligible.

Figure $4(a-h)$ is the original the auricle image and the operator the result of detection using this algorithm and the several results have been binarized, and removed the noise segments of length less than 10. As can be seen from the experimental results that the proposed algorithm has the following advantages:

(1) algorithm to extract the edge from the image illumination conditions and scale changes;

(2) Although Canny operator edge extraction is fine, but need to manually set threshold, maintain edge coherent mesh noise segment; otherwise masked the edge of some of the features;

(3) other test results of the five kinds of edge detection operators are wide edge, its coherence is not better than the proposed algorithm, and noise;

(4) the proposed algorithm to extract the characteristic curve shape is clear and complete, and fully reflect the differences of the categories included in the original image;

(5) in these types of algorithms, this algorithm is the best effect on the detection of weak edge points in the ear picture;

(6) the algorithm does not need to manually set the threshold value, can meet the next step is automatically match the identification requirements;

(7) The proposed method to extract the edge is not a single pixel, but to meet the requirements of our next recognition algorithm. If needed, the result can be refined further the e single-pixel edge of image, as shown in figure 5. 


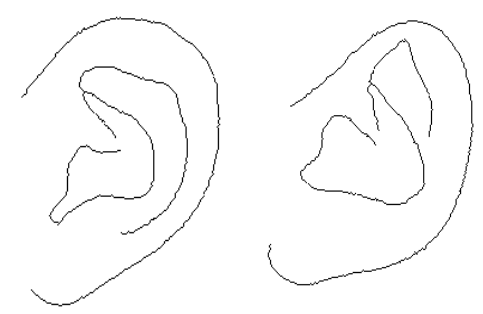

Figure 5. Thin Edge Image

\subsection{Classification and Recognition}

For a given template image and test ear image, each image can be reliably detected single pixel contour edge, and then we have to do is to classify and recognize ear image based on these feature edges.

We use Hausdorff distance(HD) of the point sets as similarity measurement of two edge images. Given two closed and bounded subsets $A$ and $B$ of a given metric space $M$., then the Hausdorff distance between $A$ and $B$ is defined as followed[26]:

$H(A, B)=\max \{h(A, B), h(B, A)\}$

Where $h(A, B)$ is so-called directed distance from $A$ to $B$. for classical Hausdorff distance:

$$
h(A, B)=\max _{a \in A} d(a, B), d(a, B)=\min _{b \in B} \rho(a, b)
$$

$d(a, B)$ is the distance from a point $a$ to the set $B$, and $\rho(a, b)$ is a point distance in the metric space $M$.

For image processing applications it has proven useful to apply a slightly different measure, the (directed) modified Hausdorff distance (MHD), which was introduced by Dubuisson et al. [27]. It is defined as:

$h_{\mathrm{mod}}(A, B)=\frac{1}{|A|} \sum_{a \in A} \min _{b \in B}\|a-b\|$

By taking the average of the single point distances, this version decreases the impact of outliers making it more suitable for pattern recognition purposes.

Solved how to evaluate the matching similarity degree of the two sections of the ear contour curve, we select one best matches the curve with curve $S$ in all known contour curve $M j$, in fact, that is to select $M j$ that its corresponding $H_{\min }$ is the minimum, as formula(8):

$H_{\text {min }}=\min \left\{H_{j}\left(M_{j}, S\right)\right\}, \quad j=1,2, \ldots, N_{m}$

Theoretically, if $H_{\min }$ is less than the pre-set threshold value $T K$, then $M j$ corresponding contour is matching.

Table 1 is the experimental results when $T K$ taken different value, In this paper, by setting $T K=70$, we obtained the maximal recognition rate. 


\section{Table 2. Matching Accuracy vs Threshold}

\begin{tabular}{ccc}
\hline Threshold(TK) & FAR & FRR \\
\hline 30 & $1.9 \%$ & $36.5 \%$ \\
40 & $2.75 \%$ & $29.2 \%$ \\
50 & $3.66 \%$ & $23.2 \%$ \\
60 & $4.57 \%$ & $13.5 \%$ \\
70 & $6 \%$ & $9.96 \%$ \\
80 & $10.7 \%$ & $7.71 \%$ \\
90 & $13.1 \%$ & $5.02 \%$ \\
\hline
\end{tabular}

Figure 6-9 is comparison our method in this paper with other methods using the same human ear experiment database. figure 6 is the result by using USTB ear database I. Figure 7 is the result by using USTB II. Figure 8 and figure 9 are the result by using USTB III. The vertical axis denotes recognition rate. It is obvious that proposed method has higher recognition rate than other algorithm in selected these three ear database.

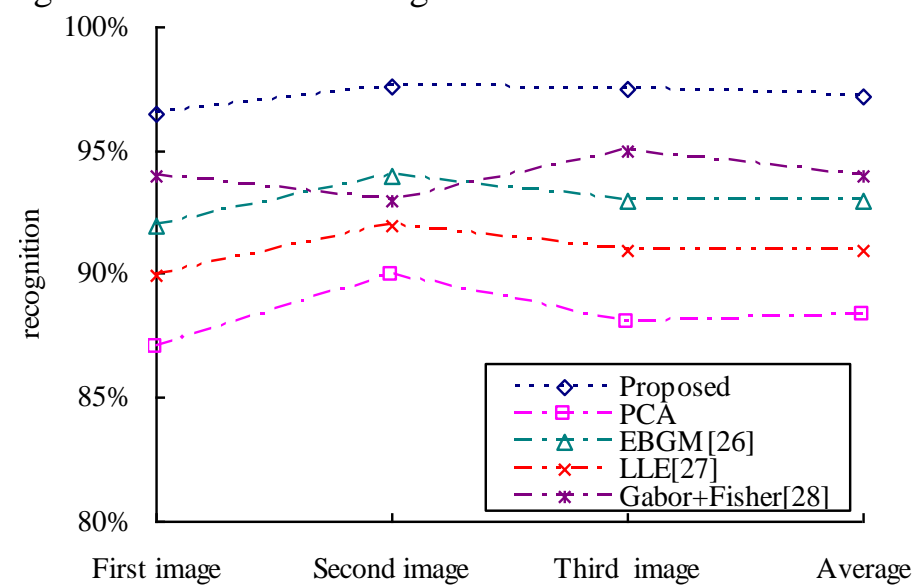

\section{Figure 6. Compare with Other Algorithm in Ear Datebase I}

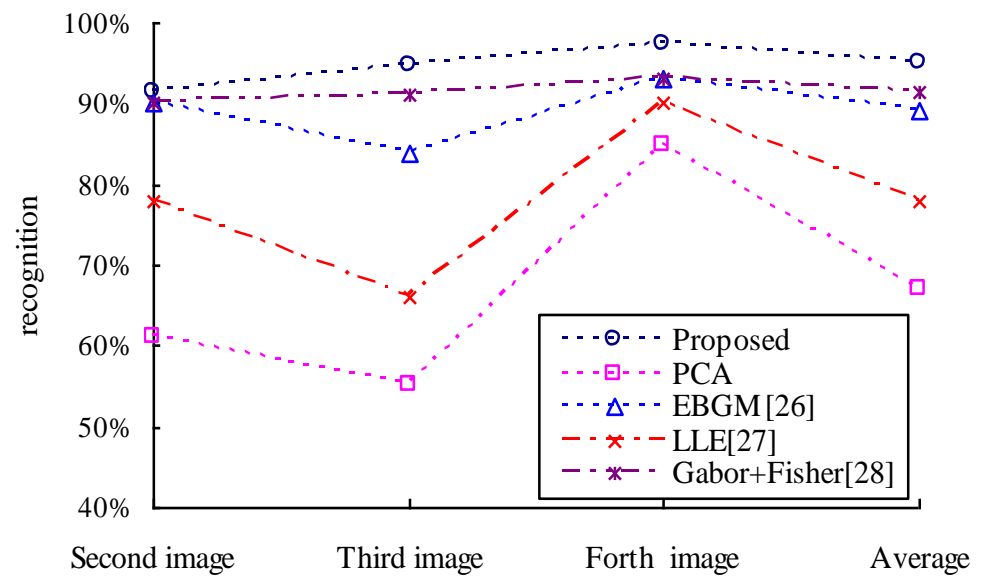

Figure 7. Compare with Other Algorithm in Ear Datebase II 


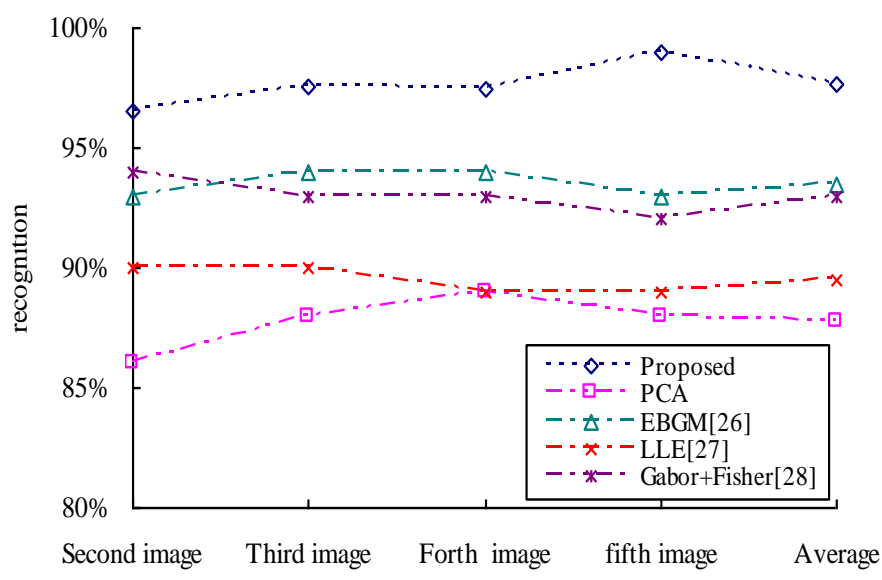

Figure 8. Compare with Other Algorithm in Ear Datebase III (Illumination Variation)

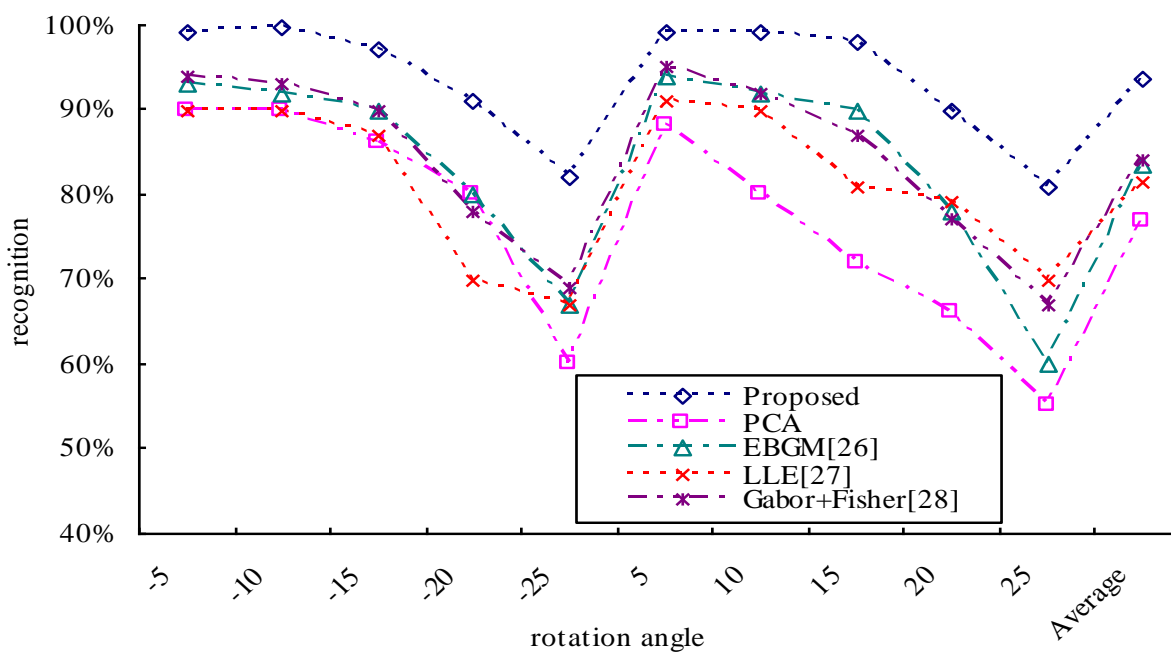

Figure 9. Compare with Other Algorithm in Ear Datebase III (Pose Variation)

\section{Conclusion}

In this paper, we presented a new characteristics of the outer ear structure based multiscale Hessian matrix edge detection method that works with edge features of grayscale images and the modified Hausdorff distance. The new method can accurately detect the the auricle anatomical feature edge point. Detected edge points are accurate, clear, good connectivity, a true reflection of the anatomical structure characteristics of the image of the original outer ear.

As a result of the application of the based on pixels Hessian matrix eigenvectors direction flow dual-threshold, not only for the local weak edge is very sensitive, and can suppress the image a gray interference and noise. Meanwhile, since the main curvature of the characteristic of the edge point is calculated in the scale space, and further enhance the stability of the feature point. In addition, this method is not required in the input image processing to set threshold value manually, and therefore better reliability for subsequent identification and determination process. Using Hausdorff distance as similarity measurement of two images, we can obtain high recognition rate in USTB ear database.

In order to improve the recognition rate, future research will concentrate on abolishing the noise and small burr on the edge curve. 


\section{Acknowledgements}

We would like to thank University of Science and Technology Beijing ear recognition Laboratory for ear image database. This paper is supported by The National Natural Science Foundation of China No.61201315 and No. 51374035.

\section{References}

[1] M. Burge and W. Burger, "Ear Biometrics for Machine Vision", 21st Workshop of the Austrian Association for Pattern Recognition (1997), pp. 275-282.

[2] W. Xiaoyun, "Study on Human Ear Recognition by Algebraic Methods". Shenyang University of Technology, ( 2011).

[3] W. Yu and X. Hong, "Application of strengthened authentication vectors to multimodal recognition of ear and face", Computer Engineering and Applications, vol.48, no.12, (2012), pp.29-33.

[4] W. Xiaoyun and G. Jinyu, "Human ear recognition based on statistic features of local information", Computer Engineering and Applications, vol. 48, no.9( 2012), pp. 141-144.

[5] J. Feng, X. Liang and Z. Mu, "Study On Ear Recognition By Using Histogram Of Oriented Gradient Features And Subregion Fuzzy Fusion".Computer Applications and Software, vol. 29, no. 4, (2012), pp. 79-82.

[6] B. Victor, K. Bowyer and S. Sarkar "An evaluation of face and ear biometrics",International Conference on Pattern Recognition, Quebec, Canada,(2002), pp. 429-432.

[7] Z. Haijun, M. Zhichun and Z. Chengyang, "Ear recognition method based on independent component analysis and BP neural network",Journal of University of Science and Technology Beijing, vol. 28, no. 6 (2006), pp. 600-603.

[8] B.Kocaman, M.Kirci and E. Gunes, "On ear biometrics".Proceedings of the IEEE 2009 International Conference on EUROCON'09, (2009), pp. 327-332.

[9] D. Jiyuan, M. Zhichun and O. Dingheng, "Multi-pose ear recognition base on a local tangent space alignment algorithm", Journal of University of Science and Technology Beijing, vol. 32, no. 12, (2010), pp. $1637-1642$.

[10] L. Xiuli, S. Hongfeng and Z. Lihua, " Ear Recognition Based on 2DLDA and FSVM". Science Technology and Engineering, no.12, (2012), pp. 2852-2855.

[11] M. Ali, M. Y. Javed and A. Basit, "Ear Recognition Using Wavelets". Proceedings of Image and Vision Computing, New Zealand,(2007), pp.83-86.

[12] L. Songze, Q. Min and W. Yi, "Human Ear Recognition Based on Gabor Wavelet and Supervised Locality Preserving Projection", Journal of Computer Aided Design \& Computer Graphics,vol. 28, no. 8 (2010), pp. 1259- 1265.

[13] L. Xiangyang, L. Ling and C. Xiaogang, "Ear Image Edge Detection Algorithm Based on Mean Shift". Journal of Information \& Computational Science, vol. 5, no .4, (2008), pp. 1765- 1770.

[14] F. Khursheed and A. H. Mir, "AR Model Based Human Identification using Ear Biometrics", International Journal of Signal Processing, Image Processing and Pattern Recognition, vol. 7, no. 3, (2014), pp. 347-360.

[15] B. Moreno and A.Sanchez, "On the Use of Outer Ear Images for Personal Identification in Security Applications ”. IEEE 33rd Annual Intl. Conf. on Security Technology, Madrid, (1999), pp. 469-476.

[16] M. Choraś, "Ear Biometrics Based on Geometrical Feature Extraction", Electronic Letters on Computer Vision and Image Analysis, vol. 5, no. 3, (2005), pp. 84-95.

[17] M. Zhichun, Y. Li and X. Zhengguang, "Shape and Structural Feature Based Ear Recognition", Advances in Biometric Person Authentication, Guangzhou, (2004), pp. 633-670.

[18] S. A. Daramola and O. D. Oluwaninyo, "Automatic Ear Recognition System using Back Propagation Neural Network". International Journal of Video \& Image Processing and Network Security, vol. 11, no. 1, (2011), pp. 28-32.

[19] M. S. Nixon, I. Bouchrika, B.Arbab-Zavar and J. N. Carter, "On use of biometrics in forensics: gait and ear", European Signal Processing Conference, Aalborg, Denmark, (2010),

[20] B. A. Zavar and M. bS. Nixon, "On guided model-based analysis for ear biometrics",Comput. Vis. Image Und., vol.11, no.5, (2011), pp. 487-502.

[21] M. Tico and P. Kuosmanen, "A topographic method for fingerprint segmentation", Proceeding of 6th International Conference on Image Processing, Kobe, Japan, (1999), pp.36-40.

[22] L. Guangming, T. Jie and Z. Mingchang, "Centerline Extraction Based on Hessian Matrix", Journal of Software, vol. 14, no. 12, (2003), pp. 2074-2081.

[23] D. G. Lowe, "Distinctive Image Features from Scale- invariant Keypoints", Intematioanal Joumal of Computer Vision, vol. 60, no. 2, (2004), pp. 91-110.

[24] M. Chi, H. Yubin and Z. Yongyong, "Fast Bi-directional SIFT Algorithm and Application", International Journal of Signal Processing, Image Processing and Pattern Recognition, vol. 7, no. 4, (2014), pp.389-398

[25] T. Ying, W. Lijun and Y. Weiqi, "New ear contour extraction method based on edge feature", Opto- 
Electronic Engineering, vol. 34, no. 4, (2007), pp. 39-43.

[26] O. Jesorsky, K. J. Kirchberg and R. W. Frischholz, "Robust Face Detection Using the Hausdorff Distance". Third International Conference on Audio-and Video-based Biometric Person Authentication, Springer, Lecture Notes in Computer Science, Halmstad,Sweden, (2001) June 6-8, pp. 90-95.

[27] M. P. Dubuisson and A.K. Jain, "A modified Hausdorff distance for object matching",In ICPR94, Jerusalem, Israel, (1994), pp. 566-568. 
International Journal of Signal Processing, Image Processing and Pattern Recognition Vol. 9, No. 5 (2016) 